\title{
Inhibition of Sonic Hedgehog Signaling Pathway by Thiazole Antibiotic Thiostrepton Attenuates the CD44+/CD24- Stem-Like Population and Sphere-Forming Capacity in Triple-Negative Breast Cancer
}

\author{
Na Yang ${ }^{a, b}$ Tai-Cheng Zhou ${ }^{a, b}$ Xiu-xia Lei ${ }^{b}$ Chang Wang ${ }^{a, c}$ Min Yan ${ }^{a}$ \\ Zi-Feng Wang ${ }^{a}$ Wei Liu ${ }^{d}$ Jian Wang ${ }^{e}$ Kai-Hua Ming ${ }^{b}$ Bi-Cheng Wang ${ }^{a}$ \\ Bang-Lao Xu ${ }^{b}$ Quentin Liu ${ }^{a}$
}

\begin{abstract}
aState Key Laboratory of Oncology in South China, Cancer Center, Collaborative Innovation Center of Cancer Medicine, Sun Yat-sen University, Guangzhou, bDepartment of Laboratory Medicine, Guangzhou First People's Hospital, Guangzhou Medical College, Guangzhou, Institute of Cancer Stem Cell, Dalian Medical University, Dalian, 'Department of Breast, Guangzhou Red Cross Hospital, Jinan University, Guangzhou, eDepartment of Pathology, GanZhou Municipal People's Hospital, NanChang University, Ganzhou, China
\end{abstract}

\section{Key Words}

Thiostrepton • Triple-negative breast cancer • Sonic hedgehog • Breast cancer stem cell

\begin{abstract}
Background/Aim: Triple-negative breast cancer (TNBC) represents a particular clinical challenge because these cancers do not respond to endocrine therapy or other available targeted agents. The lack of effective agents and obvious targets are major challenges in treating TNBC. In this study we explored the cytostatic effect of thiazole ring containing antibiotic drug thiostrepton on TNBC cell lines and investigated the molecular mechanism. Methods: Cell viability was measured by MTT assay. Cell surface marker was monitored by FCM. Western blot was applied to assess the protein expression levels of target genes. Results: We found that thiostrepton remarkably suppressed the CD44+/CD24- stem-like population and sphere forming capacity of TNBC cell lines. Notably, we showed for the first time that thiostrepton exerted its pharmacological action by targeting sonic hedgehog $(\mathrm{SHH})$ signaling pathway. Thiostrepton repressed SHH ligand expression and reduced Gli-1 nuclear localization in TNBC cell line. Furthermore, the downstream target of $\mathrm{SHH}$ signaling undergone dose-dependent, rapid, and sustained loss of mRNA transcript level after thiostrepton treatment. Finally, we showed that SHH ligand was essential for maintaining CD44+/CD24- stem-like population in TNBC cell line. Conclusion: We conclude that thiostrepton suppresses the CD44+/CD24stem-like population through inhibition of $\mathrm{SHH}$ signaling pathway. Our results give a new

N. Yang, T.-C. Zhou, X.-x. Lei and C. Wang contributed equally to this work.

Quentin Liu and Bang-Lao Xu

KARGER

State Key Laboratory of Oncology in South China, Cancer Center, Collaborative Innovation Center of Cancer Medicine Sun Yat-sen University, Guangzhou 510060 (China); Department of Laboratory Medicine, Guangzhou First People's Hospital Guangzhou Medical College, Guangzhou, (China); E-Mail liuq9@mail.sysu.edu.cn, E-Mail banglaoxu@163.com
\end{abstract}




\section{Cellular Physiology \\ Cell Physiol Biochem 2016;38:1157-1170 and Biochemistry

Yang et al.: Thiostrepton Reduces CD44+/CD24-Population by Inhibiting SHH Pathway

insight into the mechanism of thiostrepton anti-tumor activity and suggest thiostrepton as a promising agent that targets hedgehog signaling pathway in TNBC.

\section{Introduction}

Breast cancer is a highly heterogeneous malignancy that can be grouped into major subtypes. Triple-negative breast cancer (TNBC) is a distinct clinical subtype and accounts for $\sim 15 \%-20 \%$ of breast cancer patients [1]. It is defined as tumors that lack expression of estrogen (ER), progesterone (PR), and human epidermal growth factor receptor 2 (HER2). Gene expression analysis demonstrates that the molecular signature of TNBC generally overlaps with basal-like breast cancer (BLBC), with concordance rates of $70-90 \%$ [2]. TNBC often associates with more aggressive behavior, poorer prognosis, and a shorter median time to relapse and death than other forms of breast cancer. Despite the progress achieved in breast cancer therapeutic innovations the TNBC still represents a particular clinical challenge because these cancers do not respond to endocrine therapy or other available targeted agents [3]. The lack of effective agents and obvious targets are major challenges in treating patients with TNBC.

Thiostrepton, a thiazole ring containing antibiotic, originally isolated from Streptomyces azureus, has been used in veterinary medicine in mastitis caused by gram-negative organisms and in dermatologic disorders. Despite of its antibiotic action, thiostrepton has been shown to induce cell death in a variety of human cancer cell lines. In melanoma cells, thiostrepton efficiently inhibits cell growth and induces apoptosis [4]. In breast cancer cells thiostrepton induces cell cycle arrest and cell death through down-regulating FOXM1 expression [5]. Wang et al. reported that micelle-encapsulated thiostrepton reduced the growth rates of MDA-MB-231 breast cancer xenografts and HepG2-luc liver cancer xenografts [6]. Although thiostrepton has been shown to inhibit cell growth and suppress the expression of FOXM1 in breast cancer, the mechanism of its anticancer activity and its cellular targets remain undetermined.

TNBC is poorly differentiated basal-like adenocarcinomas and display breast cancer stem cell (BCSC) phenotype CD44+/CD24- [7]. The population of CD44+/CD24- cell has been demonstrated to have tumor-initiating properties and associate with stem cell-like characteristics in breast cancer [8]. Tumors with a high percentage of CD44+/CD24- cells develop significantly more distant metastases than did other tumors. Studies have revealed that the sonic hedgehog $(\mathrm{SHH})$ signaling pathway mediates progression from a non-invasive phenotype to an invasive phenotype in breast cancer. In a clinical breast cancer sample analysis, the expression of SHH and Gli-1 were significantly associated with increased risk of metastasis, breast cancer-specific death and a basal-like phenotype [9]. Moreover, SHH signaling pathway plays a key role in mammary stem/progenitor cell self-renewal by directly activating the expression of BMI-1, a central regulator of self-renewal in cancer stem cells $[10,11]$. Given the results of clinical analysis and the observation that TNBC often displays BCSC phenotype, inhibition of SHH signaling pathway is expected to be an attractive pharmacological target in TNBC $[12,13]$.

Here, we show that the thiazole ring containing antibiotic drug thiostrepton decreases the CD44+/CD24- stem-like population through inhibition of SHH signaling pathway. For the first time, we provide evidence that inhibition of SHH signaling pathway is a pharmacological action of thiostrepton. Using in vitro suspension cell culture systems, we demonstrate that thiostrepton effectively suppresses the proliferation of breast cancer stem cell of TNBC cell lines. Furthermore, we show that SHH ligand plays an important role in maintaining CD44+/ CD24- population of TNBC cell line, suggesting the crucial role of SHH signaling in TNBC. Our results provide evidence for using the antibiotic drug thiostrepton as a potential effective agent that targets SHH signaling pathway in TNBC treatment. 


\section{Cellular Physiology \\ Cell Physiol Biochem 2016;38:1157-1170 \\ \begin{tabular}{l|l}
\hline DOI: 10.1159/000443066 & (c) 2016 The Author(s). Published by S. Karger AG, Base \\
\hline
\end{tabular} and Biochemistry Published online: March 11, 2016 www.karger.com/cpb}

Yang et al.: Thiostrepton Reduces CD44+/CD24-Population by Inhibiting SHH Pathway

\section{Materials and Methods}

Cell viability and colony formation assay

MDA-MB-231, BT549, MCF-7, and T47D cells were grown in DMEM media (Invitrogen) and 10\% fetal bovine serum (Hyclone). SUM149 cells were grown in HAM'S/F12 media and 5\% fetal bovine serum, supplemented with insulin and hydrocortisone. Cells were cultured in a humidified incubator at $37^{\circ} \mathrm{C}$ with $5 \%$ CO2. Thiostrepton (Sigma) was dissolved in DMSO at a stocking concentration of $10 \mathrm{mM}$. MTT assay was used to measure cell viability as described previously [14]. Morphologic change was monitored by phasecontrast microscopy. For colony formation assay, $5 \times 10^{3}$ cells were plated in triplicate in 6 -cm plate. Twentyfour hours later, treatment was initiated. After 10 days, cells were fixed and stained with crystal violet. The number of colonies was counted using Adobe Photoshop.

\section{Flow cytometric analysis}

After treatment of thiostrepton for 24h, cells were trypsinized, washed and suspended in $100 \mu \mathrm{L}$ PBS containing preconjugated primary antibodies: CD44-FITC (1:50, eBioscience); CD24-PE(1:20, eBioscience) and incubated for $30 \mathrm{~min}$ on ice. Unstained cells were used for negative control. Cells that only stained with CD44-FITC were used to regulate compensation and set the CD24-PE gate. Cells that only stained with CD24-PE were used to regulate compensation and set CD44-FITC gate. For analysis, cells were suspended in $500 \mu \mathrm{L}$ of PBS buffer and fluorescence was measured using flow cytometer (Beckman).

\section{Mammosphere culture}

Mammosphere culture was performed as previously described [15]. Briefly, cells were seeded in ultra-low-attachment six-well plates (Corning, USA) and cultured in suspension (1000 cells/mL) in serum-free DMEM/F12 media, supplemented with B27 (1:50; Invitrogen), $20 \mathrm{ng} / \mathrm{mL}$ human recombinant epidermal growth factor (Preprotech), $20 \mathrm{ng} / \mathrm{ml}$ basic fibroblast growth factor, $4 \mu \mathrm{g} / \mathrm{ml}$ heparin and $5 \mu \mathrm{g} /$ $\mathrm{mL}$ insulin (Sigma). 5\% matrigel (BD) was added to culture medium to prevent aggregation. The number of Mammospheres were photographed and counted $10 \mathrm{~d}$ after thiostrepton treatment using inverted microscope. The diameters of the mammospheres were calculated using the Image pro plus 6.0 software.

\section{Immunofluorescence assay}

To study Gli-1 subcellular localization by immunofluorescence assay, MDA-MB-231 cells were seeded on coverslips placed in $35 \mathrm{~mm}$ dishes and treated with thiostrepton at 5 or $10 \mu \mathrm{M}$ for $24 \mathrm{~h}$, after which cells were harvested by fixing with $4 \%$ paraformaldehyde. Fixed cells were treated with anti-Gli-1 antibody (1:200, Abcam) and then the fluorochrome-tagged secondary antibody (1:200, Alexa fluor 546-conjugated anti-rabbit). Following incubation in PBS with propidium iodide, coverslips were mounted on slides.

\section{Plasmids and Transfections}

The human SHH over-expression construct was purchased from Santa Cruz Biotechnology (sc400225-ACT). For transfections, cells were seeded to a confluency of $60 \%$ and incubated for $6 \mathrm{~h}$ with a mix of antibiotic-free transfection reagents containing Lipo2000 (Invitrogen) and the plasmid DNA. Fresh medium was then added to replace the transfection reagent and cells were allowed to grow overnight before use.

\section{Small interfering RNA and Western blot}

Two human SHH siRNA oligos and control siRNA oligos were purchased from Genepharma Inc. Western blot analysis was used to confirm the knockdown of SHH expression in MDA-MB-231 cells. Cell pellets were resuspended in ice-cold RIPA lysis buffer and Western blot analyses were performed as previously described [14]. Membranes were incubated with antibodies against sonic Hedgehog ligand (Cell Signaling Technology, Inc.), Gli-1(Cell Signaling Technology, Inc.), Nanog (Epitomic, Inc.), and GAPDH (Epitomic, Inc.) at $4^{\circ} \mathrm{C}$ overnight. Protein bands were visualized with enhanced chemiluminescence.

\section{Statistical methods}

The data of this article are represented as mean \pm SD taken over a minimum of three independent experiments. Statistical significance was measured using standard t tests for two paired samples or twoway ANOVA. P values $<0.05$ were considered to be statistically significant. 


\section{Cellular Physiology \\ Cell Physiol Biochem 2016;38:1157-1170 and Biochemistry \\ Yang et al.: Thiostrepton Reduces CD44+/CD24-Population by Inhibiting SHH Pathway}

\section{Results}

Thiostrepton effectively inhibits proliferation of triple-negative breast cancer cell lines

Thiostrepton has been reported to have cytostatic effect on breast cancer cells [5, 6]. We were interested to examine whether the effect was different among different subtype of breast cancer. Three TNBC cell lines: SUM149, MDA-MB-231, BT549 and two ER-positive (ER+) breast cancer cell lines: MCF-7, T47D were treated with increasing dose of thiostrepton from 0 to $20 \mu \mathrm{M}$ over a time course of $24 \mathrm{~h}$. After $24 \mathrm{~h}$ treatment TNBC cell lines showed obvious morphologic change (Fig. 1A). MTT assay showed that after $24 \mathrm{~h}$ treatment there was an obvious reduction in proliferation in all of cell lines tested at dose of $\geq 10 \mu \mathrm{M}$. Notably the three TNBC cell lines were significantly more sensitive to thiostrepton treatment than two ER+ cell lines (Fig. 1B). Since ER+ cell lines were slow growing cells, we extended the treatment for 7 days and observed that at the dose of $5 \mu \mathrm{M}, 10 \mu \mathrm{M}$, and $15 \mu \mathrm{M}$ TNBC cell lines were also significantly more sensitive than ER+ cell lines. Nevertheless at a concentration of $20 \mu \mathrm{M}$ the cell viability of all the cell lines tested displayed sharply reduction and there was no significant difference between TNBC cell lines and ER+ cell lines (Fig. 1B). The IC50 value of ER+ cell lines were nearly or above two-fold higher than TNBC cell lines after $24 \mathrm{~h}$ treatment, whereas after 7 days treatment the difference of IC50 value between ER+ and TNBC cell lines was diminished (Table 1). These results indicate that thiostrepton induces faster cell death in TNBC cell line and it needs higher concentration and longer time to inhibit the proliferation of ER+ cell lines. Drug sensitivity was also tested by colony formation assay. Cells were plated at low density and treated continuously with $5 \mu \mathrm{M}$ or $10 \mu \mathrm{M}$ of thiostrepton for 10 days. As shown by the quantification of the colony formation assay, treatment of thiostrepton resulted in dramatically inhibition of colony formation both in TNBC cell lines and ER+ cell lines (Fig. 1C). It suggests that after long term treatment ER+ cell lines are just as sensitive as TNBC cell lines or at least that the stem-like cells within these populations may have similar sensitivity. Nevertheless, the different results between MTT assay and colony assay may be due to the reason that the higher density of more differentiated ER+ cell line are potentially less sensitive to thiostrepton. Taken together, these findings suggest that thiostrepton is a potent agent that effectively targets TNBC cell lines.

Thiostrepton reduces the CD44+/CD24- stem-like population in TNBC cell lines

Previous study has shown that TNBC often has a greater stem cell-like phenotype and a higher proportion of CD44+/CD24- breast cancer stem cell population [7]. We analyzed the two surface makers in TNBC cell lines. In consistent with previous study, MDA-MB-231 and SUM149 cells were enriched for CD44+/CD24- cells, with more than $90 \%$ and $70 \%$ respectively expressing this surface phenotype (Fig. 2A). Based on the results that TNBC cells were highly sensitive to thiostrepton treatment, we then investigated if thiostrepton had effect on the CD44+/CD24- stem cell-like population in TNBC cells. SUM149 and MDAMB-231 cells were treated with thiostrepton at $10 \mu \mathrm{M}$ and $20 \mu \mathrm{M}$ concentrations for $24 \mathrm{~h}$, after which cells were examined for stem cell marker expression. In vitro thiostrepton treatment of TNBC cells caused a significant decrease in the proportion of CD44+/CD24- cells in a concentration dependent manner (Fig. 2A). In the meantime, CD24+ cells were increased to $45.1 \%$ and $22.0 \%$ at the dose of $20 \mu \mathrm{M}$ respectively in SUM149 and MDA-MB-231 cells (Fig.

Table 1. The IC50 value of different cell line by treatment with Thiostrepton. Breast cancer cell lines were treated with increasing dose of thiostrepton from 0 to $20 \mu \mathrm{M}$ over a time course of $24 \mathrm{~h}$ or 7 days. IC50 values were calculated from the growth-inhibitory curves

\begin{tabular}{lcc}
\hline Cell line & 24h IC50 $(\mu \mathrm{M})$ & 7 Days IC50 $(\mu \mathrm{M})$ \\
\hline SUM149 & 3.82 & 2.90 \\
BT549 & 4.74 & 3.03 \\
MDA-MB-231 & 6.32 & 3.14 \\
MCF-7 & 12.25 & 5.14 \\
T47D & 13.58 & 3.96 \\
\hline
\end{tabular}




\section{Cellular Physiology Cell Physiol Biochem 2016;38:1157-1170 \begin{tabular}{l|l|l|l} 
DOI: 10.1159/000443066 & O 2016 The Author(s). Published by S. Karger AG, Basel \\
\hline
\end{tabular}

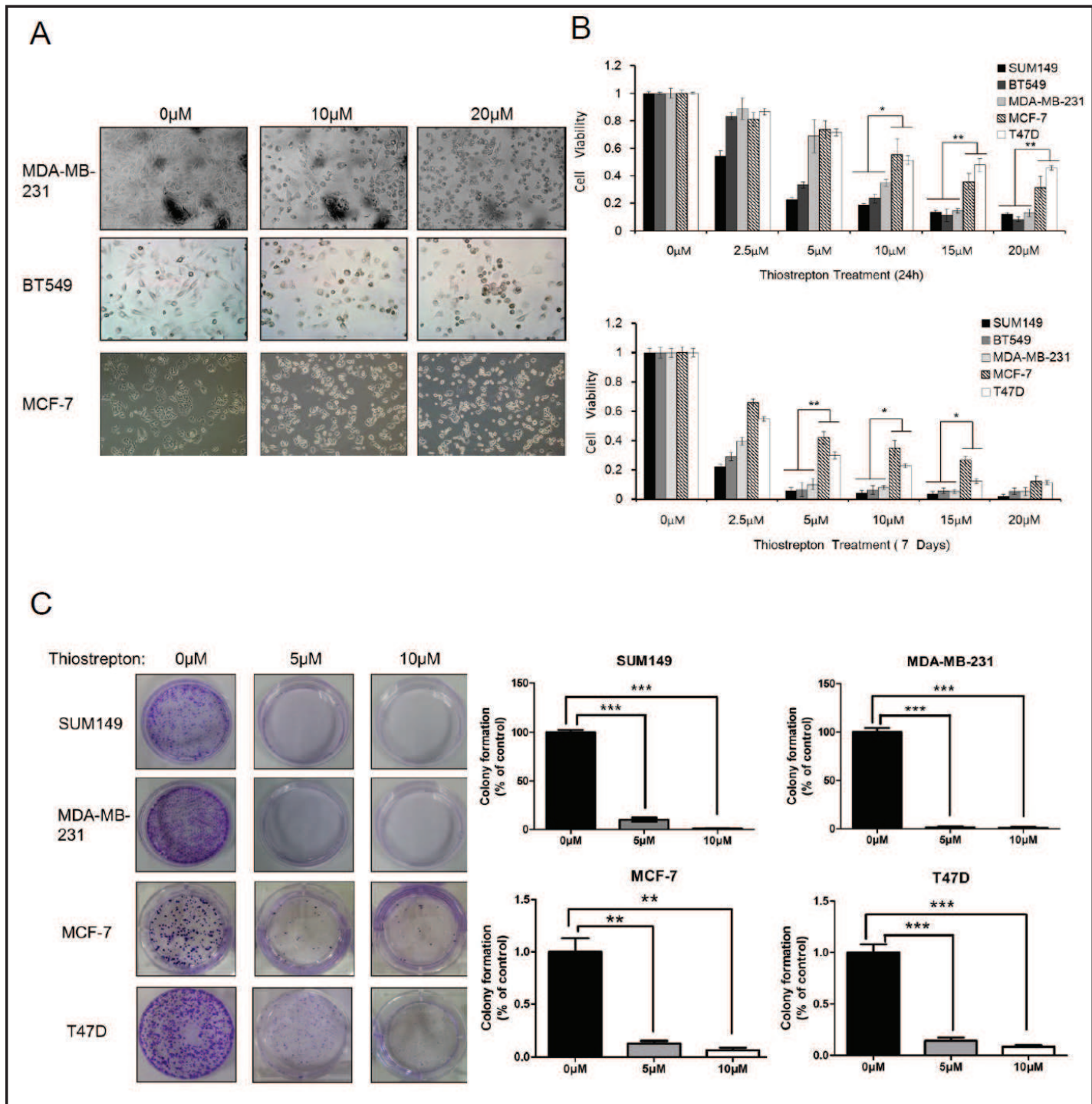

Fig. 1. The effect of thiostrepton on the proliferation of breast cancer cell lines. (A) The morphologic change of MDA-MB-231, BT549, and MCF-7 cells following 24h treatment with 0, 10, $20 \mu \mathrm{mol} / \mathrm{L}$ thiostrepton. (B) MDA-MB-231, SUM149, BT549, MCF-7, and T47D cell lines were treated with thiostrepton (0 - $20 \mu \mathrm{mol} / \mathrm{L})$ for 24 h or 7 days. Cell viability was determined by MTT assay. (C) The sensitivity of cells to thiostrepton was tested by colony-forming assay. Cell lines were treated continuously with $0,5,10 \mu \mathrm{M}$ thiostrepton for 10 days; media was changed every 3 days. Each experiment was carried out in triplicate. Columns, mean; bars, \pm SD. ${ }^{*} \mathrm{P}<0.01,{ }^{* *} \mathrm{P}<0.01,{ }^{* * *} \mathrm{P}<0.001$. Representative wells were shown.

2B). The phenomenon that CD24- cells were induced into CD24+ cells following thiostrepton treatment indicated that CD24- cells were sensitive to thiostrepton treatment.

Thiostrepton suppresses mammosphere formation of TNBC cell lines

To determine whether the loss of the stem cell marker expression actually reflected loss of stemness associated with differentiation, we employed in vitro suspension culture system to examine the effects of thiostrepton on mammosphere formation. The TNBC cell lines were seeded in suspension culture media. Primary mammospheres were grown in suspension for 7 to 10 days, after which primary mammospheres were dissociated into single cells and seeded in non-adherent culture system for more 10 days in the presence 


\section{Cellular Physiology \\ Cell Physiol Biochem 2016;38:1157-1170 \\ \begin{tabular}{l|l}
\hline DOI: 10.1159/000443066 & (c) 2016 The Author(s). Published by S. Karger AG, Base \\
\hline
\end{tabular} and Biochemistry Published online: March 11, 2016 www.karger.com/cpb

A
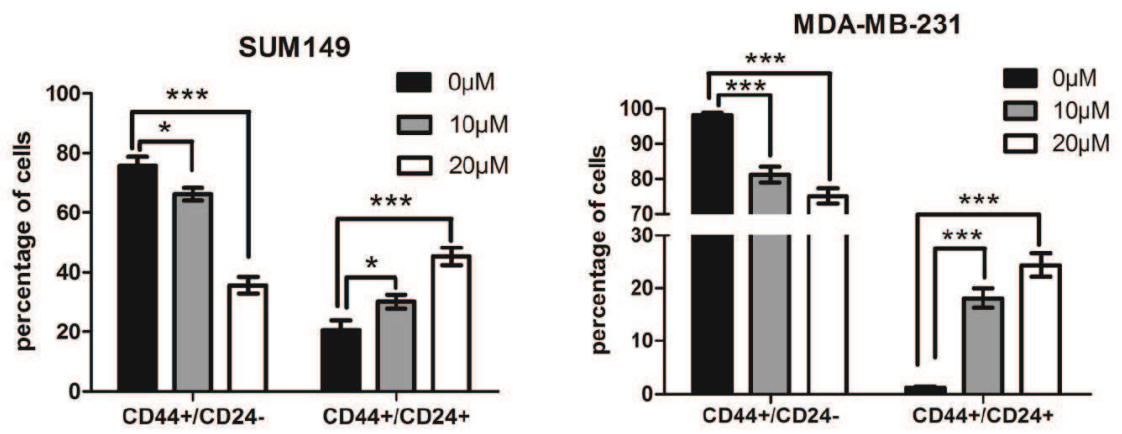

B
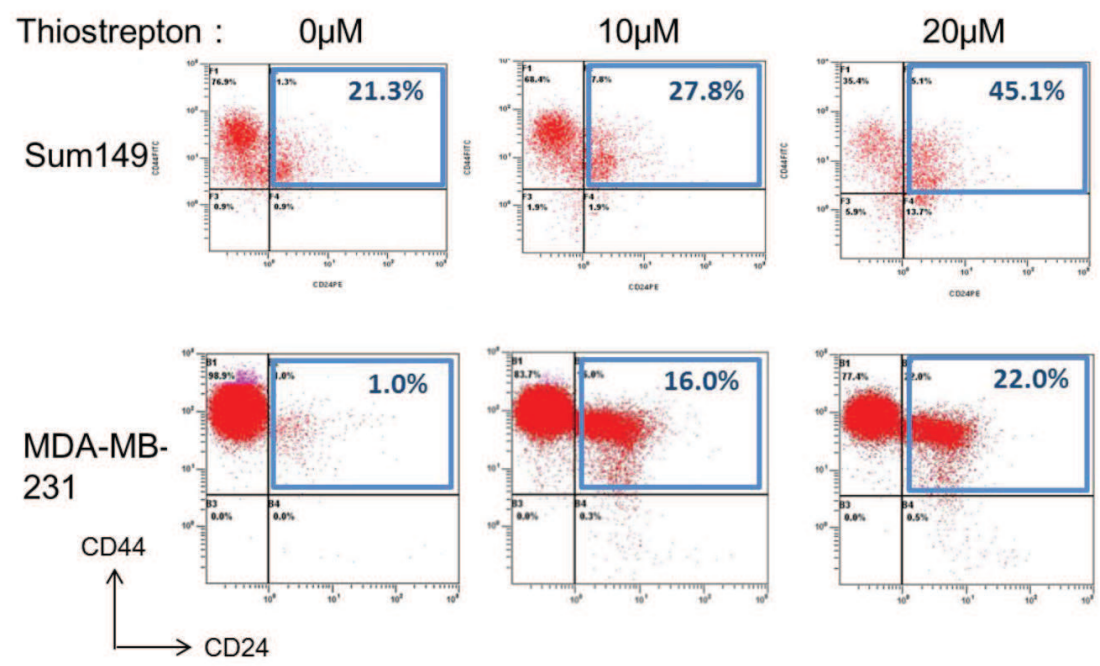

Fig. 2. Treatment of thiostrepton on the stem cell marker expression of triple-negative breast cancer cell lines. Flow cytometric analysis for cell surface phenotypes (CD44/CD24) was done in SUM149 and MDA-

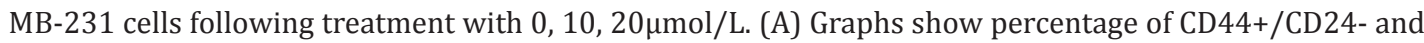
CD44-/CD24+ cells in two cell lines. Columns, mean; bars, \pm SD. ${ }^{*} \mathrm{P}<0.05$, ${ }^{* *} \mathrm{P}<0.001$. (B) Representative flow cytometric images were shown.

or absence of thiostrepton. As shown in Fig. 3A and 3B, Thiostrepton treatment caused a dramatic reduction in the number and size of mammospheres (Fig. 3C). As mammospheres are enriched for cancer stem cells, these results suggest that thiostrepton inhibits the growth of BCSC which results in significantly repressed mammosphere formation in TNBC cell lines.

Thiostrepton inhibits sonic hedgehog signaling pathway in triple-negative breast cancer cell lines

Previous studies have reported that SHH signaling plays a critical role in the regulation of CSC $[16,17]$. In breast cancer, SHH ligand activation or Gli-1 expression promotes selfrenewal of mammary stem cells [10]. Given the phenomenon that thiostrepton significantly reduced BCSC population, we hypothesized that SHH signaling pathway might be regulated by thiostrepton. To test this hypothesis, we analyzed SHH ligand and Gli-1 expression in different breast cancer cell lines. SHH ligand was highly expressed in two TNBC cell lines except for BT549, reflecting a different activation mode of SHH signaling (Fig. 4A). SUM149 


\section{Cellular Physiology \\ Cell Physiol Biochem 2016;38:1157-1170 \\ \begin{tabular}{l|l|l} 
DOI: 10.1159/000443066 & $\begin{array}{l}\text { @ 2016 The Author(s). Published by S. Karger AG, Basel } \\
\text { www.karger.com/cpb }\end{array}$ \\
\hline
\end{tabular} \\ Yang et al.: Thiostrepton Reduces CD44+/CD24-Population by Inhibiting SHH Pathway}

Fig. 3. Effects of thiostrepton on secondary mammosphere formation of TNBC cell lines. Monolayer cells were enzymatically disaggregated to a single-cell suspension and plated in nonadherent culture medium. Primary mammospheres were grown in suspension for 10 days, after which primary mammospheres were dissociated into single cells and seeded in nonadherent culture system for more 10 days in the presence or absence of thiostrepton. (A) The box plot graph shows the mammospheres size data from three independent experiments. (B) The number of mammospheres was counted and percentage of MFU (mammosphere forming unit) number was calculated. Columns, mean; bars, \pm SD. ${ }^{* * *} \mathrm{P}<0.001$. (C) Representative images were shown, Bar: $100 \mu \mathrm{m}$.

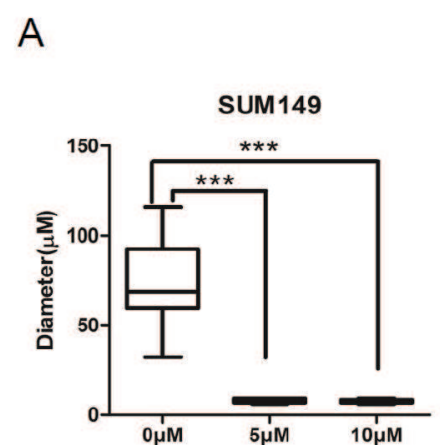

B
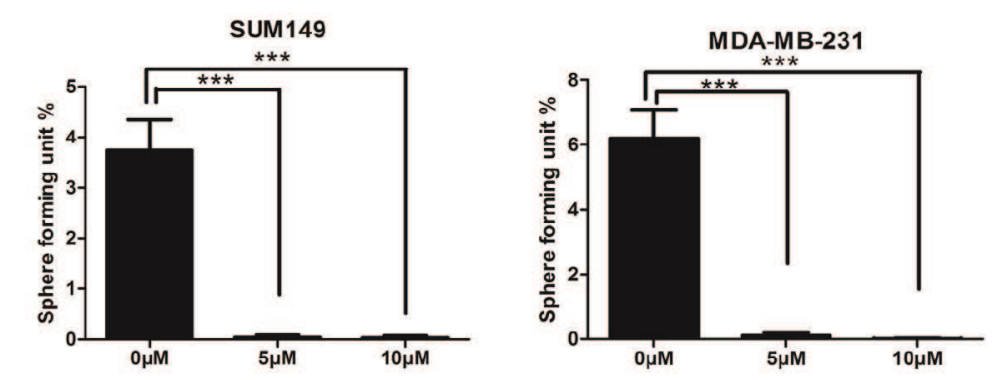

C

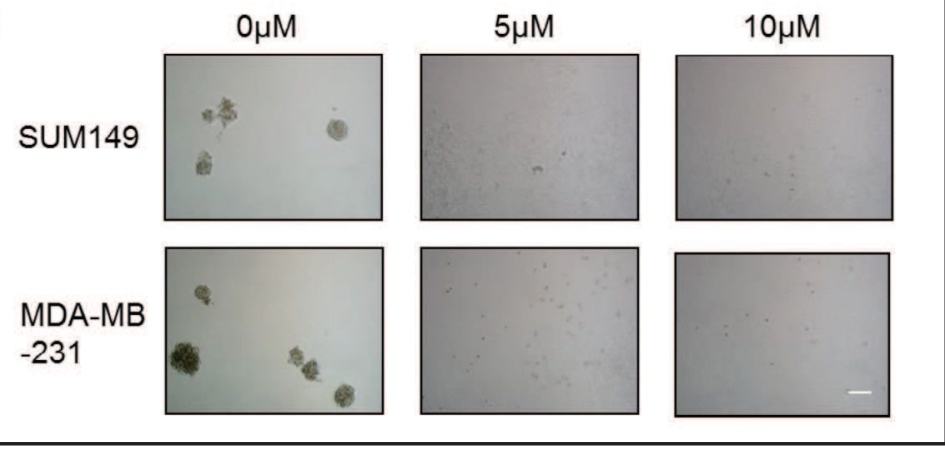

and MDA-MB-231 cells were treated with increasing doses of thiostrepton from $0-20 \mu \mathrm{M}$ for 24 h. As shown in Fig. 4B and 4C, FOXM1, a known thiostrepton target, was down-regulated upon treatment, indicative of active pharmacological action of thiostrepton. There was a pronounced decrease in SHH level after thiostrepton treatment (Fig. 4B and 4C). A significant SHH reduction was observed at a concentration of $10 \mu \mathrm{M}$, suggesting that SHH could be one of the primary cellular targets of thiostrepton. Moreover, we found that the level of nanog, a key regulator of stem cell, was obviously down-regulated by thiostrepton treatment. It is notable that there were no significant changes in the level of Gli-1, a downstream transcription factor of Hh signaling. To further validate the inhibition effects on the SHH signaling by thiostrepton, the expression of SHH signaling targeted gene was measured in MDA-MB-231 cells. Cells were treated with increasing doses of thiostrepton from 0 to $20 \mu \mathrm{M}$ for $24 \mathrm{~h}$ before harvesting for Real-time PCR analysis (Fig. 4D). The expression of FOXM1, N-MYC, and CCND1, all well-known downstream target of Hh signaling, significantly decreased in a dose dependent manner upon treatment. Previous study reported that Gli-1 nuclear location was considered a valuable marker of $\mathrm{Hh}$ pathway activation. We were curious to determine if Gli1 localization was influenced by thiostrepton. Gli-1 was mainly nuclear before treatment and became cytoplasmic after thiostrepton treatment (Fig. 4E). This result further validated the inhibition effect on SHH signaling pathway by thiostrepton. Together these results suggest 


\section{Cellular Physiology Cell Physiol Biochem 2016;38:1157-1170 \begin{tabular}{c|l|l} 
DOI: 10.1159/000443066 & O 2016 The Author(s). Published by S. Karger AG, Basel \\
and Biochemistry Published online: March 11, 2016 & wwr.com/cpb
\end{tabular}}

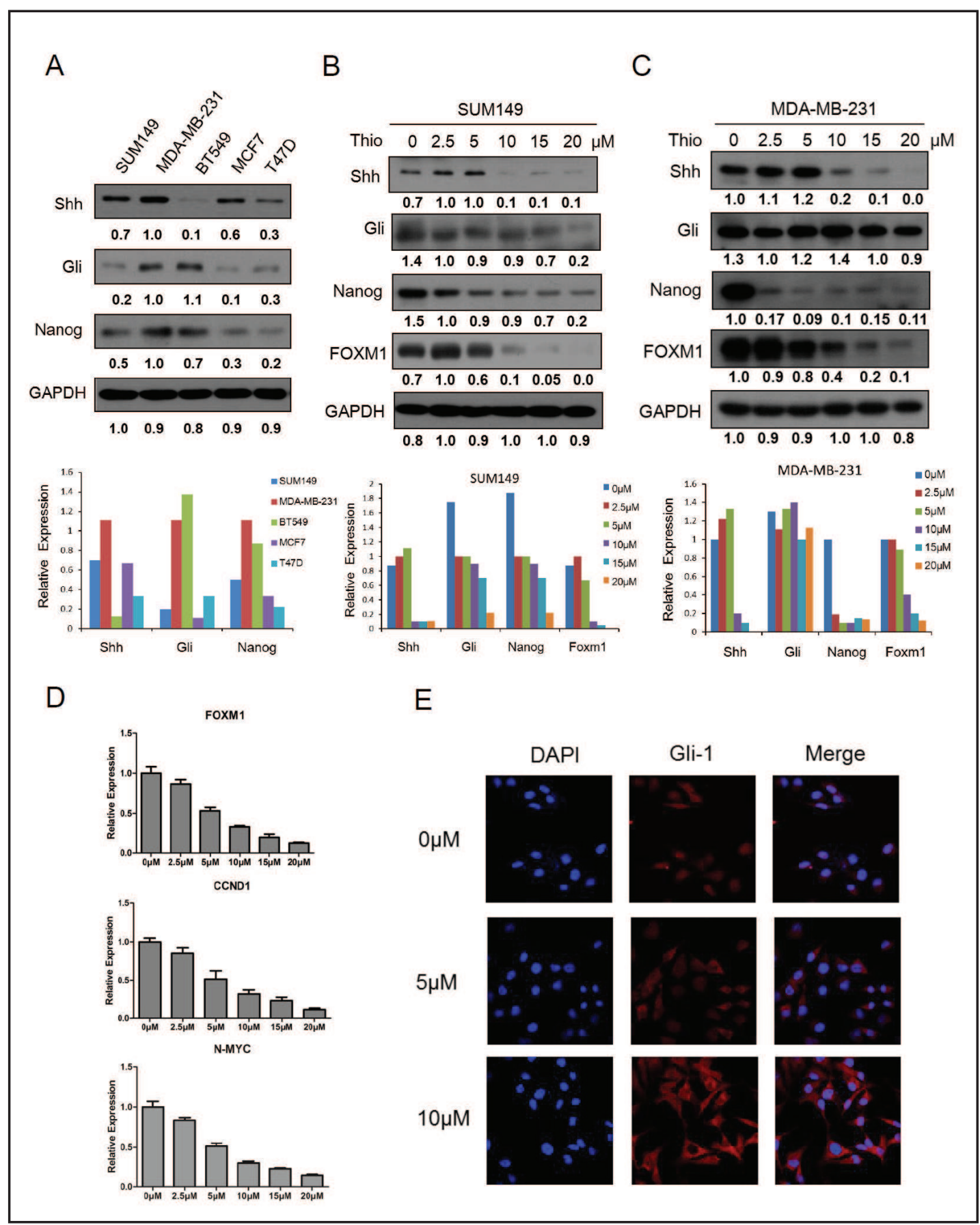

Fig. 4. Thiostrepton inhibits SHH signaling pathway in triple-negative breast cancer cell lines. (A) Protein lysates were prepared from breast cancer cell lines grown at $80 \%$ confluency in plate. Expression levels of indicated proteins were analyzed by western blotting. Densitometric evaluation was performed by image J software and represented as a histogram. (B, C) Breast cancer cell lines were treated with thiostrepton at the indicated concentration for $24 \mathrm{~h}$, after which protein lysates were prepared and analyzed by western blotting using specific antibodies. Densitometric evaluation was performed by image J software and represented as a histogram. (D) MDA-MB-231 cells were treated with indicated concentration of thiostrepton (0 - 20 $\mu \mathrm{mol} / \mathrm{L} ; 24 \mathrm{~h})$, after which mRNA was isolated and determined by RTQ-PCR with specific primer. (E) MDA-MB-231 cells were treated with $5 \mu \mathrm{M}$ or $10 \mu \mathrm{M}$ of thiostrepton, after which immunofluorescence assay was applied to detect Gli-1 staining with specific antibody. Propidium iodide was used to detect nuclear DNA. Merged images of Gli-1 and nuclear staining were also shown. 


\section{Cellular Physiology \\ Cell Physiol Biochem 2016;38:1157-1170 and Biochemistry

Yang et al.: Thiostrepton Reduces CD44+/CD24-Population by Inhibiting SHH Pathway

inhibition of SHH signaling pathway is at least one of the important pharmalogical actions of thiostrepton.

Thiostrepton decreases CD44+/CD24- stem-like population via down-regulation of SHH ligand

In the above experiment, we found that thiostrepton inhibited SHH signaling pathway and reduced CD44+/CD24- stem-like population in TNBC. Hitherto, the role of SHH ligand in maintaining CD44+/CD24- stem-like population of TNBC cells has not yet been established, we then sought to assess the effect of silencing of SHH ligand on CD44 and CD24 expression and mammosphere forming ability. Double strand SHH-antisense siRNA and control siRNA were transfected in MDA-MB-231 cells. As anticipated, SHH ligand level was decreased in antisense-expressing cells compared to negative control. Western blot analysis showed that SHH knockdown reduced the level of nanog (Fig. 5A), a well-known genes involved in CSC self-renewal, indicating that SHH ligand might modulate BCSC self-renewal through the regulation of key factors responsible for the function of CSC. To validate the functional role of SHH ligand in BCSC, we performed in vitro mammosphere formation assay. The result showed that siRNA of SHH resulted in a significant reduction in mammosphere forming unit (MFU) (Fig. 5B, $\mathrm{P}<0.01$ ). Moreover the stem cell marker analysis showed that the CD24+ cell population were increased to $14.1 \%$ (si-SHH-1) and $15.9 \%$ (si-SHH-2) in siRNA cell line compared with control cell line (Fig. 5C). To further examine the potential role of SHH ligand in the pharmacological action of thiostrepton, we over-expressed SHH ligand in MDA-MB-231 cells. The expression level of the ectopic SHH ligand remained relatively constant in the overexpression cell line compared to parental cell line in which SHH ligand was down-regulated after $10 \mu \mathrm{M}$ thiostrepton treatment (Fig. 5D). Over-expression of SHH ligand significantly prevented thiostrepton-mediated loss of cell viability by $33.7 \%(5 \mu \mathrm{M})$ and $27.4 \%(10 \mu \mathrm{M})$ respectively (Fig. 5E). We also observed that the effect of thiostrepton on CD44+/CD24- stem-like population was partly rescued in the SHH ligand overexpression cells (Fig. 5F). In conclusion, these results indicate that SHH pathway plays an important role in the maintenance of CD44+/CD24- stem-like population in TNBC cell lines. The downregulation of $\mathrm{SHH}$ ligand is at least one of the important mechanism that leads to attenuated CD44+/CD24- stem-like population.

\section{Discussion}

In this study we reported for the first time that thiazole antibiotic thiostrepton suppressed $\mathrm{CD} 44+/ \mathrm{CD} 24-$ stem-like population and sphere-forming capacity through targeting SHH signaling pathway in TNBC cell lines. Notably, we found that thiostrepton inhibited SHH signaling pathway through down-regulation of SHH ligand and reduction of nuclear Gli-1. Furthermore, we showed that SHH ligand was important for maintaining CD44+/CD24- stem-like population in TNBC cell lines.

TNBC is reported to have characteristics of more frequent recurrence, chemoresistance and shorter survival than ER+ breast cancer $[18,19]$. As a result, TNBC patients are unlikely to benefit from currently available targeted systemic therapy [20]. Strikingly, we found that the thiazole antibiotic thiostrepton effectively inhibited the cell growth of TNBC cell lines. After $24 \mathrm{~h}$ exposure to thiostrepton, all three TNBC cell lines undergone sharply reduced cell viability compared to two ER+ cell lines. When cells were treated for 7 days the growth of TNBC and ER+ cell lines were both greatly inhibited. These results indicate that thiostrepton induces faster cell death in TNBC cell line and thiostrepton is an effective agent that targeted TNBC cells.

Cancer cells from TNBC often display a profile of cell surface markers that similar to that of BCSC, characterized by the phenotype CD44+/CD24- in which CD44 is expressed at high levels but levels of CD24 are low or undetectable [21,22]. The unsatisfactory response of the TNBC to current chemotherapy and their intimate link with the CD44+/CD24- phenotype 


\section{Cellular Physiology Cell Physiol Biochem 2016;38:1157-1170 and Biochemistry POI: 10.1159/000443066 102016 The Author(s). Published by S. Karger AG, Basel

A

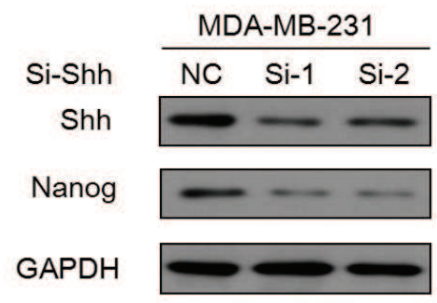

C
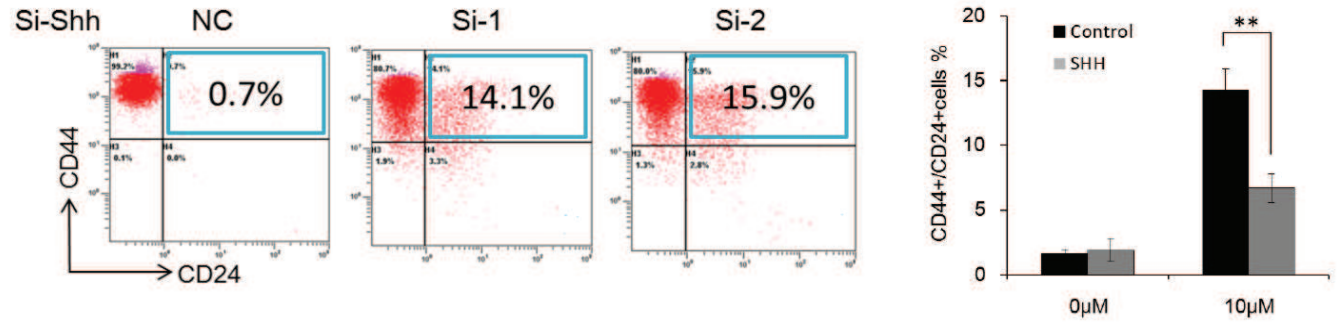

D

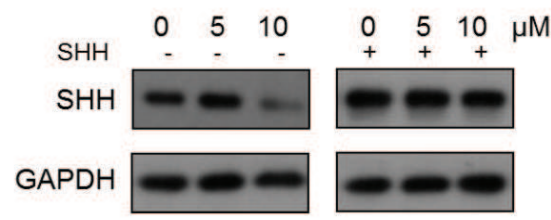

$\mathrm{F}$

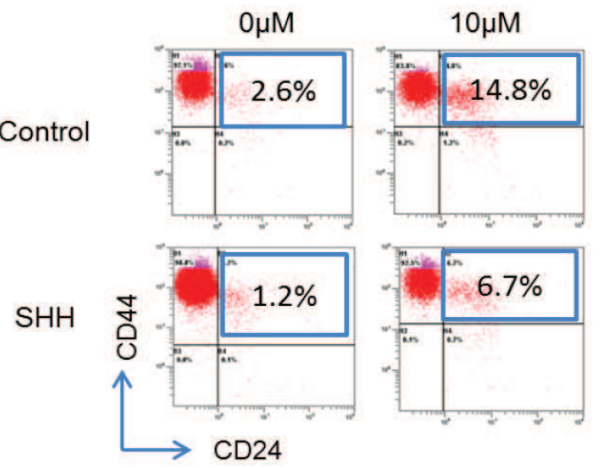

B

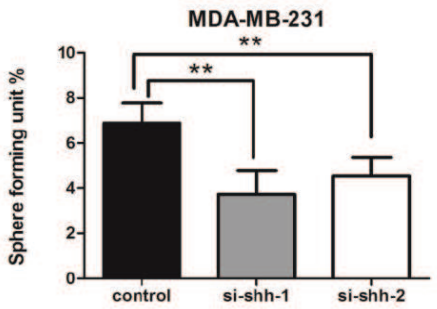

$E$
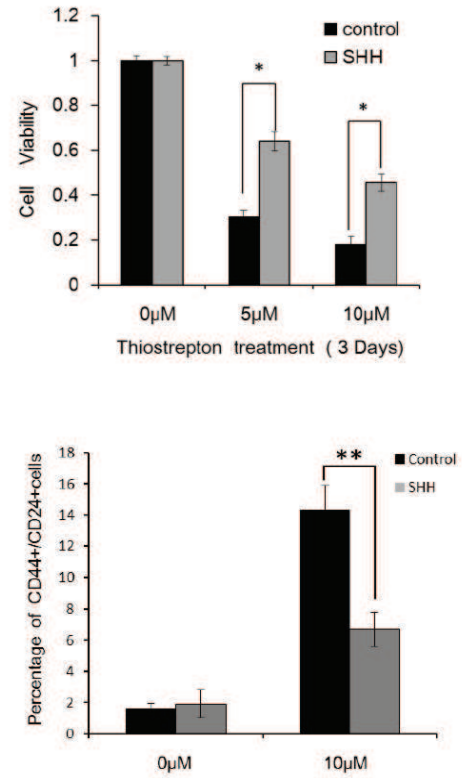

Fig. 5. Gene silencing and overexpression of SHH ligand in MDA-MB-231. (A) SHH ligand was silenced by two target RNA sequences and one negative control sequence in MDA-MB-231 cells. Western blot was used to confirm silencing effects. (B) After treated by two siRNA sequences or negative control, MDA-MB-231 cells were tested by sphere forming assay. (C) After treatment as B, MDA-MB-231 cells were analyzed for cell surface marker (CD44/CD24) using Flow cytometric analysis. (D) MDA-MB-231 cells were transfected with SHH ligand overexpression (+) or control vector (-). Both cells were treated with thiostrepton for $24 \mathrm{~h}$ at the dose of $5 \mu \mathrm{M}$ or $10 \mu \mathrm{M}$. The expression of SHH ligand was confirmed by western blot. GAPDH was used as loading control. (E) After treatment as D, cell viability was determined by MTT assay. (F) After treatment as D, cell surface marker (CD24/CD44) was analyzed by Flow cytometric analysis. Representative flow cytometirc images are shown. Each experiment was carried out in triplicate. Columns, mean; bars, \pm SD. $* \mathrm{P}<0.05,{ }^{*} \mathrm{P}<0.01$. 


\section{Cellular Physiology Cell Physiol Biochem 2016;38:1157-1170 \\ \begin{tabular}{ll|l} 
and Biochemistry.1159/000443066 & $\begin{array}{l}\text { C) } 2016 \text { The Author(s). Published by S. Karger AG, Base } \\
\text { www.karger.com/cpb }\end{array}$ \\
\hline
\end{tabular}}

make CD44/CD24 targeting an attractive therapeutic alternative for TNBC patients. Consistent with earlier reports, we find that TNBC cell lines: SUM149 and MDA-MB-231 show more than $70 \%$ of cells expressing the CD44+/CD24- phenotype. Strikingly, in vitro thiostrepton $(20 \mu \mathrm{mol} / \mathrm{L})$ treatment of TNBC cells caused $>20 \%$ decrease in the proportion of CD44+/CD24- cells (Fig. 2A). In breast cancer, CD44 is positively associated with stem celllike characteristics and CD24 expression is related to differentiated epithelial features. The decrease of CD24- cells indicates that CD24- cells were sensitive to thiostrepton treatment. In cancer stem cell model, therapies that promote differentiation significantly improve survival $[23,24]$. It was reported that some infant tumors underwent spontaneous differentiation that leads to a favorable outcome even without therapy [25]. The observations from our group suggest that targeting differentiation marker CD24 may be an effective strategy to treat TNBC. Furthermore, thiostrepton remarkably suppressed mammosphere formation of TNBC cell lines in vitro suspension culture system, indicating that thiostrepton effectively inhibited BCSC number and activity of TNBC in vitro. Our observation that thiazole antibiotic thiostrepton effectively inhibited BCSC activity also consistent with a recent intriguing findings in which antibiotics were found to selectively target CSC in multiple tumor types [26].

Identification of specific target for TNBC patients remains an important clinical challenge. Evidence has revealed the significant roles of SHH signaling in TNBC [9, 27-29]. In a clinical sample immunohistochemistry analysis the expression of SHH ligand and Gli1 in TNBC was significantly increased in comparison to non-triple-negative breast cancer (nTNBC) [9]. Another group recapitulated the finding that SHH ligand over-expression in a mouse model of basal breast cancer increased growth and induced a poorly differentiated phenotype [30]. We analyzed SHH ligand and Gli-1 expression in different breast cancer cell lines. SHH ligand was highly expressed in two TNBC cell lines except for BT549, reflecting a different activation mode of SHH signaling. Indeed, except for autocrine, a paracrine mode of SHH signaling has also been described in the basal-like subtype of breast cancer: the nonmalignant stromal secrete SHH ligand, leading to SHH pathway activation in tumour cells. This communication between epithelial and stromal compartments plays an important role in breast cancer progression [31]. Interestingly, we found that SHH ligand protein level underwent dramatic decrease at the dose of $10 \mu \mathrm{M}$ thiostrepton treatment, suggesting that SHH ligand may be a key target of thiostrepton. Previous study reported that thiostrepton inhibited proteasome which results in the stabilization of certain proteins that proves fatal to cancer cells [32]. This is unlikely to be the global action of thiostrepton because in our study we observed not only SHH ligand but also nanog, a key regulator of stem cell, were obviously down-regulated rather than stabilized by thiostrepton. It is intriguing to investigate if SHH ligand could be also suppressed by other proteasome inhibitors, such as Siomycin A, MG132 and bortezomib, etc. in the future. The inhibition effect of thiostrepton on SHH signaling pathway was further validated by the dose-dependent, rapid, and sustained loss of mRNA transcript level of certain target genes of SHH pathway, including CCND1 and N-Myc. Hitherto, the pharmacological action of thiostrepton is not well understood. Evidence has shown that thiostrepton affects the expression of FOXM1 at the gene promoter level and not through de novo protein synthesis because thiostrepton fails to repress an ectopic FOXM1 driven by a CMV promoter [5, 33]. It is interesting to note that FOXM1 has been shown to be an important downstream transcriptional target of Gli-1 [34]. Given the observation in our study, it raises the possibility that thiostrepton suppresses SHH signaling pathway which leads to reduced FOXM1 mRNA level. Although we cannot exclude the possibility that other mechanisms have involved, our study shed light on the potential mechanism of thiostrepton on FOXM1 expression. Moreover, we find that the SHH transcription factor Gli-1 nuclear localization is reduced following thiostrepton treatment. Since Gli-1 nuclear location is considered the activation of SHH pathway [35-37], this phenomenon further validated the inhibition effect of thiostrepton on SHH signaling pathway. Taken together, our study suggested that the inhibition of SHH signaling by thiostrepton might contribute to its anticancer activity. 


\section{Cellular Physiology \\ Cell Physiol Biochem 2016;38:1157-1170 and Biochemistry

Targeting stem cell related signaling pathway is supposed to be an effective therapeutic strategy for treatment of TNBC. SHH signal pathway has been shown to play a pivotal role in the maintenance of self-renewal of mammary stem cells [10]. Activation of hedgehog signaling increases breast cancer mammosphere initiating cell number, whereas inhibition of the pathway results in a reduction of these effects. Consistent with the inhibition effect of SHH signaling pathway by thiostrepton, we find that RNA silencing of SHH ligand also attenuates the CD44+/CD24- stem-like population and sphere-forming ability (Fig. 5B and 5C). In addition, over-expression of SHH ligand partly rescues the thiostrepton-mediated loss of CD44+/CD24- population. These results suggest that SHH ligand plays a pivotal role in the maintenance of CD44+/CD24- population. Although we cannot exclude the possibility that thiostrepton suppresses CD44+/CD24- stem-like population and sphere-forming ability through an as yet unknown molecular mechanism, our results indicate that down-regulation of SHH ligand is an important mechanism that leads to decreased CD44+/CD24- population by thiostrepton treatment.

Our findings that SHH signaling pathway is targeted by thiostrepton has important therapeutic implications. To date, there are already a number of clinical trials employing Hh inhibitor, including natural compound cyclopamine and synthetic small molecules in pancreatic, colorectal and breast cancer [38]. However, possible concerns have derived from the poor knowledge of tolerance and efficacy of inhibiting these mechanisms without inducing severe side effects. Some clinical trials have been reported to be suspended owing to side effects of $\mathrm{Hh}$ inhibitor [28]. As a natural antibiotic, thiostrepton has already used in veterinary medicine and exhibits no toxicity on normal breast cancer cell line MCF-10A [5, 39]. Thus the toxic side effects of thiostrepton-based therapy would be minimized. Moreover, thiostrepton has been reported recently to be successful delivered by nanoparticles in human breast cancer xenografts models to significantly reduce tumor burden [6]. Our findings suggest that thiostrepton is efficacious for targeting TNBC cell lines through the inhibition of SHH signaling pathway. Further work is required to assess whether this is true in vivo.

Overall, we report that thiostrepton effectively suppresses cell growth and CD44+/ CD24- stem-like population of TNBC cell lines via inhibition of SHH signaling pathway. Down-regulation of SHH ligand protein level might be one of the mechanisms whereby thiostrepton exerts its cytotoxic effect on TNBC cells. We also show that SHH ligand plays an important role in the maintainance of CD44+/CD24- stem-like population in TNBC cell lines. Our results give a new insight into the mechanism of thiostrepton anti-tumor activity and provide evidence for using thiostrepton as an effective therapeutic method to treat $\mathrm{SHH}$ over-expression TNBC.

\section{Acknowledgements}

We thank other members of the Quentin Liu lab for their critical comments and technical support. We also thank Prof. Eric W-F Lam (Imperial College) for his kindly suggestion. This research work was supported by the National Basic Research Program of China (973 Program; No. 2012CB967000 to Q.L.) Sister Institution Network Fund (SINF) 2012 and, National Natural Science Foundation of China (81130040 to Q.L.).

\section{Disclosure Statement}

The authors declare no competing financial interest.

\section{References}

1 Gluz O, Liedtke C, Gottschalk N, Pusztai L, Nitz U, Harbeck N: Triple-negative breast cancer--current status and future directions. Ann Oncol 2009;20:1913-1927. 


\section{Cellular Physiology \\ Cell Physiol Biochem 2016;38:1157-1170 \\ \begin{tabular}{l|l}
\hline DOI: $10.1159 / 000443066$ & C 2016 The Author(s). Published by S. Karger AG, Basel
\end{tabular} and Biochemistry Published online: March 11, 2016 www.karger.com/cpb}

Yang et al.: Thiostrepton Reduces CD44+/CD24-Population by Inhibiting SHH Pathway

2 Rakha EA, Reis-Filho JS, Ellis IO: Basal-like breast cancer: A critical review. J Clin Oncol 2008;26:25682581.

3 Gelmon K, Dent R, Mackey JR, Laing K, McLeod D, Verma S: Targeting triple-negative breast cancer: Optimising therapeutic outcomes. Ann Oncol 2012;23:2223-2234.

4 Bhat UG, Zipfel PA, Tyler DS, Gartel AL: Novel anticancer compounds induce apoptosis in melanoma cells. Cell Cycle 2008;7:1851-1855.

5 Kwok JM, Myatt SS, Marson CM, Coombes RC, Constantinidou D, Lam EW: Thiostrepton selectively targets breast cancer cells through inhibition of forkhead box m1 expression. Mol Cancer Ther 2008;7:2022-2032.

6 Wang M, Gartel AL: Micelle-encapsulated thiostrepton as an effective nanomedicine for inhibiting tumor growth and for suppressing foxm1 in human xenografts. Mol Cancer Ther 2011;10:2287-2297.

7 Ricardo S, Vieira AF, Gerhard R, Leitao D, Pinto R, Cameselle-Teijeiro JF, Milanezi F, Schmitt F, Paredes J: Breast cancer stem cell markers cd44, cd24 and aldh1: Expression distribution within intrinsic molecular subtype. J Clin Pathol 2011;64:937-946.

8 Al-Hajj M, Wicha MS, Benito-Hernandez A, Morrison SJ, Clarke MF: Prospective identification of tumorigenic breast cancer cells. Proc Natl Acad Sci USA 2003;100:3983-3988.

9 Tao Y, Mao J, Zhang Q, Li L: Overexpression of hedgehog signaling molecules and its involvement in triplenegative breast cancer. Oncol Lett 2011;2:995-1001.

10 Liu S, Dontu G, Mantle ID, Patel S, Ahn NS, Jackson KW, Suri P, Wicha MS: Hedgehog signaling and bmi-1 regulate self-renewal of normal and malignant human mammary stem cells. Cancer Res 2006;66:60636071.

11 Cheng W, Yu P, Wang L, Shen C, Song X, Chen J, Tang F, Yang Q: Sonic hedgehog signaling mediates resveratrol to increase proliferation of neural stem cells after oxygen-glucose deprivation/reoxygenation injury in vitro. Cell Physiol Biochem 2015;35:2019-2032.

12 Kameda C, Tanaka H, Yamasaki A, Nakamura M, Koga K, Sato N, Kubo M, Kuroki S, Tanaka M, Katano M: The hedgehog pathway is a possible therapeutic target for patients with estrogen receptor-negative breast cancer. Anticancer Res 2009;29:871-879.

13 Ramaswamy B, Lu Y, Teng KY, Nuovo G, Li X, Shapiro CL, Majumder S: Hedgehog signaling is a novel therapeutic target in tamoxifen-resistant breast cancer aberrantly activated by pi3k/akt pathway. Cancer Res 2012;72:5048-5059.

14 Wu X, Yang N, Zhou WH, Xu J, Chen JJ, Zheng FM, Long ZJ, Yue CF, Ai KX, Liu LL, Wan XY, Liu Q: Up-regulation of p21 inhibits trail-mediated extrinsic apoptosis, contributing resistance to saha in acute myeloid leukemia cells. Cell Physiol Biochem 2014;34:506-518.

15 Zheng FM, Long ZJ, Hou ZJ, Luo Y, Xu LZ, Xia JL, Lai XJ, Liu JW, Wang X, Kamran M, Yan M, Shao SJ, Lam EW, Wang SW, Lu G, Liu Q: A novel small molecule aurora kinase inhibitor attenuates breast tumor-initiating cells and overcomes drug resistance. Mol Cancer Ther 2014;13:1991-2003.

16 Merchant AA, Matsui W: Targeting hedgehog--a cancer stem cell pathway. Clin Cancer Res 2010;16:31303140 .

17 Tanaka H, Nakamura M, Kameda C, Kubo M, Sato N, Kuroki S, Tanaka M, Katano M: The hedgehog signaling pathway plays an essential role in maintaining the $\operatorname{cd} 44+\mathrm{cd} 24-/$ low subpopulation and the side population of breast cancer cells. Anticancer Res 2009;29:2147-2157.

18 Engebraaten 0, Vollan HK, Borresen-Dale AL: Triple-negative breast cancer and the need for new therapeutic targets. Am J Pathol 2013;183:1064-1074.

19 Ferguson LL, Curran B, Martinez M, Mancuso P: Triple-negative breast cancer: What is known about it? Clin J Oncol Nurs 2014;18:E6-E11.

20 Andreopoulou E, Schweber SJ, Sparano JA, McDaid HM: Therapies for triple negative breast cancer. Expert Opin Pharmacother 2015;16:983-998.

21 Foulkes WD, Smith IE, Reis-Filho JS: Triple-negative breast cancer. N Engl J Med 2010;363:1938-1948.

22 Honeth G, Bendahl PO, Ringner M, Saal LH, Gruvberger-Saal SK, Lovgren K, Grabau D, Ferno M, Borg A, Hegardt C: The cd44+/cd24- phenotype is enriched in basal-like breast tumors. Breast Cancer Res 2008;10:R53.

23 Magee JA, Piskounova E, Morrison SJ: Cancer stem cells: Impact, heterogeneity, and uncertainty. Cancer cell 2012;21:283-296. 


\section{Cellular Physiology \\ Cell Physiol Biochem 2016;38:1157-1170 \\ \begin{tabular}{l|l}
\hline DOI: $10.1159 / 000443066$ & (c) 2016 The Author(s). Published by S. Karger AG, Basel
\end{tabular} and Biochemistry Published online: March 11, 2016 www.karger.com/cpb}

Yang et al.: Thiostrepton Reduces CD44+/CD24-Population by Inhibiting SHH Pathway

24 Diederichs S, Zachert K, Raiss P, Richter W: Regulating chondrogenesis of human mesenchymal stromal cells with a retinoic acid receptor-beta inhibitor: Differential sensitivity of chondral versus osteochondral development. Cell Physiol Biochem 2014;33:1607-1619.

25 Baker DL, Schmidt ML, Cohn SL, Maris JM, London WB, Buxton A, Stram D, Castleberry RP, Shimada H, Sandler A, Shamberger RC, Look AT, Reynolds CP, Seeger RC, Matthay KK, Children's Oncology G: Outcome after reduced chemotherapy for intermediate-risk neuroblastoma. N Engl J Med 2010;363:1313-1323.

26 Lamb R, Ozsvari B, Lisanti CL, Tanowitz HB, Howell A, Martinez-Outschoorn UE, Sotgia F, Lisanti MP: Antibiotics that target mitochondria effectively eradicate cancer stem cells, across multiple tumor types: Treating cancer like an infectious disease. Oncotarget 2015;6:4569-4584.

27 Kasper M, Jaks V, Fiaschi M, Toftgard R: Hedgehog signalling in breast cancer. Carcinogenesis 2009;30:903911.

28 Hui M, Cazet A, Nair R, Watkins DN, O'Toole SA, Swarbrick A: The hedgehog signalling pathway in breast development, carcinogenesis and cancer therapy. Breast Cancer Res 2013;15:203.

29 Kubo M, Nakamura M, Tasaki A, Yamanaka N, Nakashima H, Nomura M, Kuroki S, Katano M: Hedgehog signaling pathway is a new therapeutic target for patients with breast cancer. Cancer Res 2004;64:60716074.

30 Harris LG, Pannell LK, Singh S, Samant RS, Shevde LA: Increased vascularity and spontaneous metastasis of breast cancer by hedgehog signaling mediated upregulation of cyr61. Oncogene 2012;31:3370-3380.

31 O'Toole SA, Machalek DA, Shearer RF, Millar EK, Nair R, Schofield P, McLeod D, Cooper CL, McNeil CM, McFarland A, Nguyen A, Ormandy CJ, Qiu MR, Rabinovich B, Martelotto LG, Vu D, Hannigan GE, Musgrove EA, Christ D, Sutherland RL, Watkins DN, Swarbrick A: Hedgehog overexpression is associated with stromal interactions and predicts for poor outcome in breast cancer. Cancer Res 2011;71:4002-4014.

32 Pandit B, Bhat UG, Gartel AL: Proteasome inhibitory activity of thiazole antibiotics. Cancer Biol Ther 2011;11:43-47.

33 Bhat UG, Halasi M, Gartel AL: Foxm1 is a general target for proteasome inhibitors. PLoS One 2009;4:e6593.

34 Teh MT, Wong ST, Neill GW, Ghali LR, Philpott MP, Quinn AG: Foxm1 is a downstream target of gli1 in basal cell carcinomas. Cancer Res 2002;62:4773-4780.

35 Li Y, Yang W, Yang Q Zhou S: Nuclear localization of gli1 and elevated expression of foxc2 in breast cancer is associated with the basal-like phenotype. Histol Histopathol 2012;27:475-484.

36 Cho HK, Kim SY, Kim KH, Kim HH, Cheong J: Tumor suppressor protein vhl inhibits hedgehog-gli activation through suppression of gli1 nuclear localization. FEBS Lett 2013;587:826-832.

37 Xu J, Huang G, Zhang Z, Zhao J, Zhang M, Wang Y, Liu Z, Lu J: Up-regulation of glioma-associated oncogene homolog 1 expression by serum starvation promotes cell survival in er-positive breast cancer cells. Cell Physiol Biochem 2015;36:1862-1876.

38 Drenkhahn SK, Jackson GA, Slusarz A, Starkey NJ, Lubahn DB: Inhibition of hedgehog/gli signaling by botanicals: A review of compounds with potential hedgehog pathway inhibitory activities. Curr Cancer Drug Targets 2013;13:580-595.

39 Tsang SW, Zhang HJ, Chen YG, Auyeung KK, Bian ZX: Eruberin a, a natural flavanol glycoside, exerts antifibrotic action on pancreatic stellate cells. Cell Physiol Biochem 2015;36:2433-2446. 